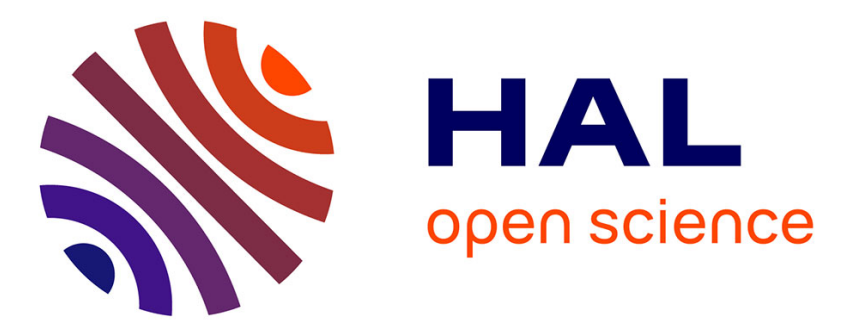

\title{
Relative behaviour of sound absorption and dispersion near the nematic-smectic-A phase transition in TBBA and CBOOA : comment and new results
}

P. Martinoty

\section{- To cite this version:}

P. Martinoty. Relative behaviour of sound absorption and dispersion near the nematic-smectic-A phase transition in TBBA and CBOOA : comment and new results. Journal de Physique Lettres, 1979, 40 (13), pp.291-296. 10.1051/jphyslet:019790040013029100 . jpa-00231627

\section{HAL Id: jpa-00231627 \\ https://hal.science/jpa-00231627}

Submitted on 1 Jan 1979

HAL is a multi-disciplinary open access archive for the deposit and dissemination of scientific research documents, whether they are published or not. The documents may come from teaching and research institutions in France or abroad, or from public or private research centers.
L'archive ouverte pluridisciplinaire HAL, est destinée au dépôt et à la diffusion de documents scientifiques de niveau recherche, publiés ou non, émanant des établissements d'enseignement et de recherche français ou étrangers, des laboratoires publics ou privés. 


\title{
Relative behaviour of sound absorption and dispersion near the nematic-smectic-A phase transition in TBBA and CBOOA : comment and new results
}

\author{
P. Martinoty \\ Laboratoire d'Acoustique Moléculaire (*), Université Louis-Pasteur, 4, rue Blaise-Pascal, 67070 Strasbourg, France
}

(Reçu le 25 avril 1979, accepté le 16 mai 1979)

\begin{abstract}
Résumé. - Nous comparons les résultats obtenus par ultrasons au voisinage de la transition nématique-smectique-A dans le TBBA et le CBOOA. Nous montrons que les anomalies dans la vitesse et dans l'atténuation peuvent être expliquées par une relaxation de la chaleur spécifique. Nous discutons les contributions de cisaillement, les absorptions résiduelles et les régimes en fréquences.
\end{abstract}

\begin{abstract}
Ultrasonic results obtained in TBBA and CBOOA near the nematic-smectic-A phase transition are compared. In both compounds the anomalies in the velocity and in the attenuation can be explained by a relaxation of the heat capacity. The critical shear contributions, the background absorptions, and the frequency regimes are discussed.
\end{abstract}

1. Introduction. - The critical anomalies exhibited by sound absorption and dispersion have long been recognized as important for the study of dynamic critical phenomena [1].

In the field of liquid crystals, ultrasonic measurements near the nematic-isotropic $(\mathrm{N}-\mathrm{I})$ phase transition showed a pronounced maximum in the attenuation and a minimum in the velocity. (For a recent study of the N-I transition, see ref. [2] and references cited therein.)

At the smectic-A-nematic (A-N) phase transition, however, ultrasonic measurements are rather scarce [3-9] and the current situation appears confusing. This letter is an attempt to clarify this situation.

The study that Kiry and I made of the A-N transition in CBOOA [6-8] revealed that this transition is characterized by weak pretransitional effects. Our analysis above $T_{\mathrm{AN}}$ [8] showed that the ultrasonic absorption has two components. The dominant one comes from the excess specific heat due to the fluctuations. This component gives rise to a diverging bulk viscosity, $\delta \eta$, which causes an isotropic increase in the attenuation. The other component is due to the critical increase in the shear viscosity, $v_{1}$ in the notation of ref. [11]. Since $\delta \eta \sim\left(\delta c_{p}\right) \tau_{\mathrm{m}}$ [8] and $v_{1} \sim \tau_{\mathrm{m}} / \xi$ [10], the temperature dependence of the two compo-

(*) E.R.A. au C.N.R.S. nents is very different. Here $\tau_{\mathrm{m}}$ is the relaxation time of the order parameter, $\xi$ is the correlation length, and $\delta c_{p}$ is the excess specific heat.

In a recent paper [9], Bhattacharya, Sarma and Ketterson observed that TBBA, unlike CBOOA, has large anomalies in the velocity and the attenuation. In their opinion, the larger anomalies in TBBA reflect a more-second-order nature of the A-N transition.

However, reported volumetric, calorimetric, and NMR data (summarized in table I) show unambiguously that the A-N transition is closer to first-order in TBBA than in CBOOA. Thus the large anomalies in the velocity and the attenuation in TBBA do not indicate a more strongly second-order nature of the A-N transition. In this respect one must note that these anomalies are still larger at the N-I transition, which is by nature first-order.

One aim of the present paper is to discuss the relative importance of the pretransitional anomalies in velocity and attenuation near the A-N and N-I transitions on the basis of the dynamic heat capacity theory. As we shall see below, the theory explains the isotropic anomalies observed by Bhattacharya et al. in TBBA and permits interpretation of the relative behaviours of sound absorption and dispersion in TBBA and CBOOA.

The paper also discusses other aspects of the results in the two compounds such as the background absorption and the shear contribution. In TBBA, 
Table I. - Summary of results showing that the A-N transition is more first-order in TBBA than in CBOOA. Results at the N-I transition are shown for comparison.

\begin{tabular}{|c|c|c|c|c|}
\hline Systems & $\begin{array}{l}\Delta T \\
{ }^{\circ} \mathrm{C}\end{array}$ & $\begin{array}{c}\Delta H \\
(\mathrm{cal} / \mathrm{g})\end{array}$ & $\begin{array}{l}10^{4} \Delta \bar{V} \\
\left(\mathrm{~cm}^{3} / \mathrm{g}\right)\end{array}$ & $\begin{array}{l}\text { Orientational } \\
\text { order parameter }\end{array}$ \\
\hline - & - & - & - & - \\
\hline TBBA (A-N) & $\sim 1\left(^{a}\right)$ & $0.17\left(^{b}\right)$ & $40\left(^{a}\right)$ & jump $\left({ }^{c}\right)$ \\
\hline CBOOA $(A-N)$ & $0.08 \pm 0.04\left(^{d}\right)$ & $\begin{array}{r}0.06\left(^{d}\right) \\
<0.02\left(^{f}\right)\end{array}$ & $\begin{array}{l}0.5 \pm 0.2\left(^{d}\right) \\
\quad<0.1\left(^{f}\right)\end{array}$ & no jump $\left({ }^{e}\right)$ \\
\hline PAA (N-I) & & $0.68\left(^{g}\right)$ & $35\left(^{h}\right)$ & \\
\hline
\end{tabular}

$\Delta T$ is the width of the transition observed by volumetric studies; $\Delta H$ is the transition enthalpy and $\Delta \bar{V}$ is the volume jump.

$\left({ }^{a}\right)$ Unpublished results from D. Guillon; $\left(\left(^{b}\right)\right.$ ref. [21]; $\left(^{c}\right)$ ref. [22]; $\left({ }^{d}\right)$ ref. [23]; $\left({ }^{e}\right)$ ref. [24]; $\left({ }^{f}\right)$ ref. [25]; $\left({ }^{g}\right)$ ref. [26]; $\left({ }^{h}\right)$ ref. [27].

unlike CBOOA, the shear contribution is not observable. New results for TBBA are also presented showing, in particular, that the relaxation frequency is higher than that deduced by Bhattacharya et al. from the shifts in the attenuation peaks.

2. The relaxation of the specific heat. - In the dynamic heat capacity theory, only the temperature variations produced by the sound wave are considered to have a practical effect on the system, causing dispersion and absorption as in the usual thermal relaxation processes. In this theory, the critical absorption per wavelength and the velocity dispersion can be described in terms of a single reduced frequency $\omega^{*}=\omega / \omega_{0}$. Here $\omega$ is the angular frequency of the sound wave and $\omega_{0}$ is the characteristic frequency of the fluctuations. Results obtained for Xe [12-13] or for binary mixtures [14] are in good agreement with the theory in the region where $\omega^{*} \lesssim 10$. In the region of large $\omega^{*}$, there is a discrepancy between experimental and theoretical values and one must consider the effects of fluctuations whose wave numbers are much greater than $\xi^{-1}$ [15]. For CBOOA we have shown that the data may be consistently and quantitatively interpreted with this theory for

$$
T>T_{\mathrm{AN}}+0.8^{\circ} \mathrm{C},
$$

i.e., in a region where $\omega^{*}<10$. (In the notation of ref. [8], $\omega^{*}=1 / x$.)

The dispersion, $\Delta V=V(\infty)-V(0)$, the maximum value of the absorption per wavelength, $(\alpha \lambda)_{\text {Max }}$, and the ultrasonic absorption, $\alpha / f^{2}$ (in its low-frequency limit), are given by $[8,16]$ :

$$
\begin{gathered}
\frac{\Delta V}{V_{0}}=\frac{1}{2}\left(\gamma_{0}-1\right) \frac{\delta c_{p}}{c_{p}^{0}} \\
(\alpha \lambda)_{\operatorname{Max}} \simeq(0.3) \Pi\left(\gamma_{0}-1\right) \frac{\delta c_{p}}{c_{p}^{0}} \\
\frac{\alpha}{f^{2}}=\frac{2 \Pi^{2}}{V_{0}}\left(\gamma_{0}-1\right) \frac{\delta c_{p}}{c_{p}^{0}} \tau
\end{gathered}
$$

where $\delta c_{p}$ is the excess specific heat due to the fluctuations, and $c_{p}^{0}$ and $\gamma_{0}$ are respectively the specific heat at constant pressure and the specific heat ratio in the absence of fluctuations. $V(0)$ and $V(\infty)$ are the velocities of sound at zero and infinite frequencies, respectively. The acoustical relaxation time, $\tau$, that appears in eq. (3) is not the relaxation time of the order parameter $\tau_{\mathrm{m}}$, but is proportional to $\tau_{\mathrm{m}}$ $\left(\tau^{-1}=b \tau_{\mathrm{m}}^{-1}\right.$ with $\left.b \sim 8[2,8]\right)$.

On the basis of this theory the following observations can be made :

1) The dip in the sound velocity and the increase in the attenuation are both isotropic, which explains the results of Bhattacharya et al.

2) The magnitudes of the critical anomalies can be directly discussed from relations (1) and (2), which do not involve the relaxation time, $\tau$.

According to these relations, the larger the ratio $\delta c_{p} / c_{p}^{0}$, the larger the anomalies in $\Delta V / V_{0}$ and $(\alpha \lambda)_{\text {Max }}$. Since measurements of the specific heat show that the ratio $\delta c_{p} / c_{p}^{0}$ is larger in TBBA than in CBOOA, the fact that the anomalies in the velocity and attenuation are larger for TBBA than for CBOOA is also explained qualitatively (for a detailed comparison between the data see table II).

3) Although the values of $\delta c_{p}$ are larger in TBBA than in CBOOA, one cannot deduce that the transition is more strongly second-order in TBBA than in CBOOA, which is precisely the suggestion made by Bhattacharya et al. Indeed, in the context of the mean-field assumption, the excess specific heat can be written as [8]

$$
\delta c_{p}=C_{0} T^{2} \xi_{\|}
$$

where $\xi_{\|}^{2}=1 / 2 A M_{\mathrm{v}}, A=a\left(T-T_{\mathrm{AN}}\right)$, and $C_{0}$ is a constant characteristic of the compound.

Thus, the thermal variation of $\delta c_{p}$ is given by $\xi_{\|}$, but the order of magnitude of $\delta c_{p}$ depends on both $\xi_{\|}$ and $C_{0}$.

$C_{0}$ is given by [8]

$$
C_{0}=\frac{k}{16 \Pi}\left(2 a M_{\mathrm{T}}\right)\left(2 a M_{\mathrm{V}}\right)
$$

where $k$ is the Boltzmann's constant. 
Table II. - Specific heat values deduced from the ultrasonic (US) experiments and those measured by differential scanning calorimetry (DSC).

$$
\begin{gathered}
V(\infty)-V(0) \\
\hline V(0) \\
- \\
- \\
>0.02\left({ }^{c}\right) \\
>0.07\left(^{d}\right) \\
>0.15\left({ }^{f}\right)
\end{gathered}
$$

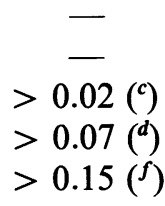$$
(\alpha \lambda)_{\operatorname{Max}}
$$

CBOOA

TBBA

MBBA

PAA

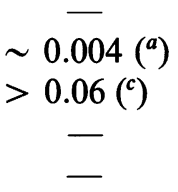<smiles></smiles>

$\sim \overline{0} .02$

$>0.32$

$>0.7$

$>1.5$<smiles></smiles><smiles>[SeH2]</smiles>

$\sim 0.03(\bar{b}) ; \sim 0.06$

$\sim 0.44$

$\sim 0.67\left({ }^{e}\right) ; \sim 0.8$

$\sim 2.2$

$\left({ }^{a}\right)$ Ref. [8]; $\left({ }^{b}\right)$ ref. [25]; $\left({ }^{c}\right)$ ref. [9]; $\left({ }^{d}\right)$ ref. [28]; $\left({ }^{e}\right)$ ref. [35]; $\left({ }^{f}\right)$ ref. [2].

The values of $\delta c_{p} / c_{p}^{0}$ are deduced from ultrasonic experiments using formulas (1) and (2) with $\gamma_{0}=1.2$.

The velocity at infinite frequency, $V(\infty)$, was estimated by extrapolating the velocity in the isotropic phase to $T_{\mathrm{c}}$, and the velocity at zero frequency, by taking the minimum value observed at the transition. The symbol $>$ signifies that our estimate is an underestimate.

The velocity dispersion is so weak for CBOOA that it could not be measured. An estimate of $V(\infty)-V(0)$ can be made using the value of $\delta c_{p} / c_{p}^{0}$ and relation (1) : we find $V(\infty)-V(0) \sim 2.5 \mathrm{~m} / \mathrm{s}$.

For TBBA, $(\alpha \lambda)_{\text {Max }}$ is not known; the value reported above is the value of $\alpha \lambda$ at $T=T_{\mathrm{AN}}$ for $10 \mathrm{MHz}$ (from Fig. 2 of ref. [9]).

An estimate of $C_{0}$ can be made using the measured values of $\xi_{\|}$at $\Delta T=1{ }^{\circ} \mathrm{C}\left(\xi_{\|}^{\text {TBBA }} \simeq 100 \AA[17]\right.$; $\left.\xi_{\|}^{\mathrm{CBOOA}} \simeq 560 \AA[18]\right)$ and assuming $M_{\mathrm{T}}=40 M_{\mathrm{V}}[18]$. One finds $C_{0}^{\mathrm{TBBA}} \simeq 10^{8}$ CGS units and $C_{0}^{\mathrm{CBOOA}} \simeq$ $10^{5}$ CGS units. Therefore the larger value of $C_{0}$ in TBBA explains the larger value of $\delta c_{p}$ and the inverse relation between $\delta c_{p}$ and $\xi_{\|}$.

A similar argument can be made for the N-I transition. Here $C_{0}$ is given by [19]

$$
C_{0}=\frac{g k}{32 \Pi}\left(\frac{a}{L}\right)^{2}
$$

where $L$ is an elastic constant and $g$ a parameter that describes the contribution of the various correlation functions.

For PAA, $g=10$ and $a / L=10^{12} \mathrm{~cm}^{-2} \mathrm{~K}^{-1}$ [19], and therefore $C_{0}=1.4 \times 10^{7}$ CGS units. This high value explains the large contribution of the specific heat to the attenuation and velocity for this compound, although the correlation length is very small $\left(\xi\left(T_{\mathrm{c}}\right) \sim 100 \AA\right)$. Since $a / L \sim 10^{12} \mathrm{~cm}^{-2} \mathrm{~K}^{-1}$ for PCB and MBBA [20], a similar conclusion can be obtained for these compounds.

Table II shows good agreement between the specific heat values deduced from the ultrasonic measurements for various compounds and those measured directly by differential scanning calorimetry.

\section{The contribution of the shear viscosity to the} absorption. - As mentioned previously, the ultrasonic absorption presents two contributions above $T_{\mathrm{AN}}$, one reflecting the behaviour of the specific heat and the other due to the increase in the friction coefficient, $v_{1}$.

In CBOOA, the anomalies in the attenuation were not isotropic, and it is possible to separate the shear contribution, which is not directly observable, by considering the following combination of the experimental data [36] :

$$
\alpha_{\|}+\alpha_{\perp}-2 \alpha_{45} \sim \delta v_{1} .
$$

Here $\alpha_{\|}, \alpha_{\perp}$, and $\alpha_{45}$ are the attenuation coefficients for $\theta=0^{\circ}, \theta=90^{\circ}$, and $\theta=45^{\circ}$, respectively, where $\theta$ is the angle between the director and the propagation of the wave.

In TBBA, Bhattacharya et al. used the same procedure to evaluate the shear contribution. However, this procedure is not adequate here since their figure 1 shows that the increase in the attenuation is isotropic above $T_{\mathrm{AN}}$. This isotropic increase is easily explained by the fact that in TBBA the high tempe-

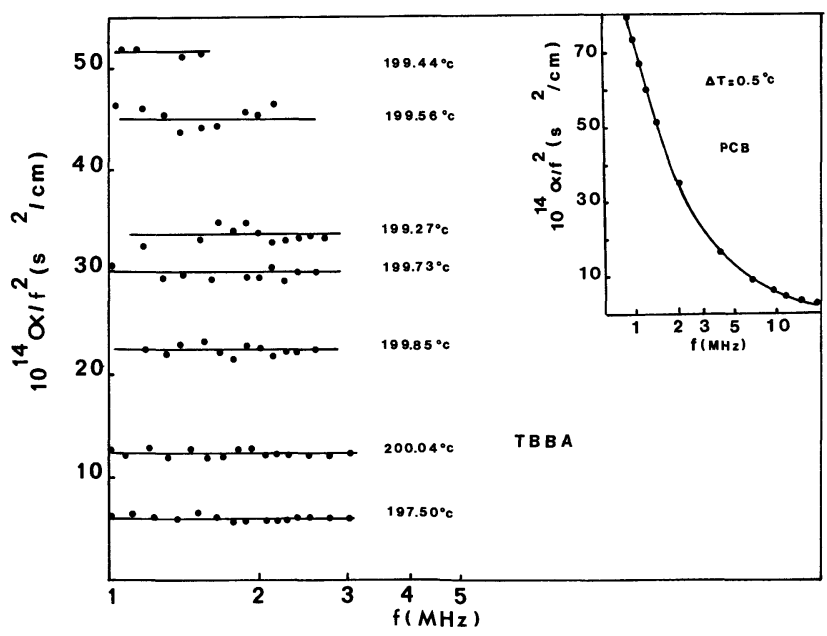

Fig. 1. - The absorption coefficient divided by the square of the frequency, as a function of frequency. Individual curves are for various temperatures above and below the transition. The transition temperature was $T_{\mathrm{AN}}=199.5 \pm 0.15^{\circ} \mathrm{C}$. This value is an average of the values found before and after the ultrasonic experiments (see text). The data show that $\alpha / f^{2}$ is frequency-independent and are within the $\omega \tau \ll 1$ regime. Results for PCB in a region where $\omega \tau \gtrsim 1$ are shown for comparison (from ref. [37]). Note the strong decrease in $\alpha / f^{2}$ between 1 and $3 \mathrm{MHz}$ for this compound. The frequency relaxation was about $1.3 \mathrm{MHz}$. 
rature of the transition makes the shear contribution very weak.

4. The background absorption. - In CBOOA the background absorption was attributed to two additive contributions, namely, a relaxation of the chains and the residual part of the N-I critical contribution.

In TBBA, Bhattacharya et al. also attributed the background absorption to the relaxation of the chains. However, their figure 1 shows that the background absorption at $2 \mathrm{MHz}$ above $T_{\mathrm{AN}}$ is abnormally high

$$
\left(\alpha / f^{2} \sim 4.500 \times 10^{-17} \mathrm{~cm}^{-1} \mathrm{~s}^{2}\right)
$$

and a relaxation of the chains analogous to that in MBBA [30] (which has a butyl chain, as does TBBA) or Merck V [29] cannot account for this value. Indeed, extrapolation of the Arrhenius plot of $\tau^{-1}$ and $(\alpha \lambda)_{\operatorname{Max}}$ obtained at room temperature for Merck V or $\mathrm{MBBA}$ to the temperature range where TBBA is nematic suggests that the flexibility of the chain cannot contribute significantly to the background absorption. The residual part of the N-I contribution and the classical absorption due to viscosities are also too small to explain the high value of the background. The aromatic skeleton of TBBA, unlike those of other liquid crystals, is a double schiff base, whose deformations were observed by NMR [31]. These deformations probably contribute to the ultrasonic absorption.

5. The frequency regime. - As emphasized by Bhattacharya et al., a knowledge of the frequency regime is important to compare the data with theories. In CBOOA our measurements showed that the frequency regime is $\omega \tau \lesssim 1\left(\omega^{*}<10\right.$ in terms of the reduced frequency). In TBBA, Bhattacharya et al. claimed that their measurements were in the regime $\omega \tau \gtrsim 1$. Their conclusion rested mainly on the observation of a shift in the position of the attenuation maximum with changing frequency.

However, from the data at the temperature farthest below $T_{\mathrm{AN}}$ in their figure 2 , one can deduce that $\alpha / f^{2}$ goes from $\sim 10.000 \times 10^{-17} \mathrm{~cm}^{-1} \mathrm{~s}^{2}$ to $1.000 \times 10^{-17} \mathrm{~cm}^{-1} \mathrm{~s}^{2}$ when the frequency goes from 2 to $10 \mathrm{MHz}$. This decrease in $\alpha / f^{2}$ with increasing frequency shows evidence of a strong relaxation effect, the relaxation frequency of which is below $10 \mathrm{MHz}$. This relaxation cannot be attributed to the critical one, since according to the law for $\tau^{-1}$ given by Bhattacharya et al., the frequency relaxation would be $\sim 70 \mathrm{MHz}$ in this temperature range. This additional relaxation (which was unknown) probably affects both the position of the attenuation peak and the scaling of its magnitude with $\omega$. This leads one to question the conclusions of Bhattacharya et al. concerning the frequency regime, especially the law for the relaxation frequency.

A direct way to determine the frequency regime is to see whether the behaviour of $\alpha / f^{2}$ is frequencydependent or frequency-independent. The results in TBBA that I obtained with this procedure are reported here.

An acoustic resonator was used. This device has the advantage of giving absolute absorption values without being calibrated, unlike the fixed-path cell used in the traditional pulse technique for the study of smectic phases. Furthermore, the resonator is very sensitive, having revealed pretransitional anomalies in CBOOA that were not revealed by the traditional technique [5]. The set-up and the experimental procedure are described in refs. [2] and [8].

In the present study, to prevent oxidation, all parts in contact with the liquid crystal were gold-plated, and the Viton ring for parallelism adjustment in figure 1 of ref. [2] was teflon-coated. After being degassed under vacuum, TBBA was introduced into the cell in an atmosphere of dry nitrogen gas; then the cell was sealed. The temperature of the cell was controlled to within $\pm 0.03^{\circ} \mathrm{C}$.

TBBA samples were prepared at the Ecole de Chimie of Strasbourg. The transition temperatures measured under a microscope were within a fraction of a degree of the values already reported [32]. Differential scanning calorimetry (DSC) showed a small peak at the A-N transition and none at the AC transition. The A-N transition temperature was measured before and after the ultrasonic experiments. In spite of the aforementioned precautions shifts of as much as $0.3^{\circ} \mathrm{C}$ were found in the transition temperature from one run to another, as also reported by Bhattacharya et al. Since it was not possible to accurately define the difference $\Delta T=\left|T-T_{\text {AN }}\right|$ close to $T_{\text {AN }}$ the results in figure 1 are given as a function of the absolute temperature $T$. Furthermore, visual observation of the light scattering under a microscope shows that the two phases coexisted over a temperature range never smaller than $0.2^{\circ} \mathrm{C}$, depending on the purity of the samples. This region of coexistence was not eliminated even after repeated distillation of the sample [5]. Other researchers have made similar observations in TBBA (see table I) and in MBBA $[30,33,34]$, which are both Schiff's bases $\left({ }^{1}\right)$. A region of coexistence, but smaller, has also been observed for CBOOA [23].

Figure 1 shows typical data for $\alpha / f^{2}$, the ultrasonic absorption, as a function of $f$, the frequency in the two phases. The striking feature in this figure is the frequency-independent behaviour of the ultrasonic absorption, even in the region of transition where the maximum value of $\alpha / f^{2}$ at $2 \mathrm{MHz}$ is of the same order of magnitude as that measured by Bhattacharya et al. The results in figure 1 show that the frequency regime is $\omega \tau \ll 1$, in contrast with the regime $\omega \tau \gtrsim 1$ found by Bhattacharya et al., and imply that the shift in the position of the attenuation peak is smaller than that reported by these authors. Note that $\alpha / f^{2}$ is also

$\left.{ }^{1}\right)$ For MBBA a region of coexistence of $0.2^{\circ} \mathrm{C}$ was noticed even for samples having clearing temperatures greater than $47^{\circ} \mathrm{C}$ [34]. 
frequency-independent for the $T$ farthest below $T_{\mathrm{AN}}$, in contrast to their results. In this region there is a strong discrepancy between the 2-MHz absorption measurements since, from figure 1 of Bhattacharya et al., $\alpha / f^{2} \sim 15.000 \times 10^{-17} \mathrm{~cm}^{-1} \mathrm{~s}^{2}$ for $\theta=90^{\circ}$, whereas we found $\alpha / f^{2} \sim 5.500 \times 10^{-17} \mathrm{~cm}^{-1} \mathrm{~s}^{2}$. The various experimental discrepancies in the absorption data between the results presented here and those of Bhattacharya, Sarma and Ketterson, could be due to the experimental problems we have already mentioned, in particular to the existence of the region where the two phases overlap, and also to the use of different ultrasonic devices, as discussed above.

6. Conclusion. - We have shown that a relaxation of the specific heat accounts for the isotropic dip in the velocity and the isotropic increase in the attenuation observed in TBBA above $T_{\text {AN }}$ by Bhattacharya, Sarma and Ketterson.

Using the dynamic heat capacity theory, we have given a qualitative understanding of the relative behaviours of the sound absorption and the velocity in TBBA and CBOOA. In particular we have shown that the magnitude of the anomalies does not indicate the first- or the second-order character of the transition.

We have also pointed out that CBOOA has a critical increase in the attenuation that is not isotropic. This is caused by the shear contribution which is large for this compound because of its relatively low transition temperature. In contrast, TBBA has a critical increase in the attenuation that is isotropic, and the shear contribution cannot be observed for this compound. Its high transition temperature may also explain why its frequency-regime $(\omega \tau \ll 1)$ is different from that of CBOOA $(\omega \tau \sim 1)$ for the same ultrasonic frequency range near $T_{\mathrm{AN}}$.

In the context of liquid crystals the simple model provided by the dynamic heat capacity theory offers a convenient framework for experimenters since it gives an estimate of the critical anomalies in the velocity and attenuation from the data of a routine technique such as differential scanning calorimetry. Of course, this theory is not sufficient to explain more complicated behaviour that might appear at temperatures very close to $T_{c}$, such as mode-coupling between the order parameter and the hydrodynamic shear mode [38].

Acknowledgments. - I am indebted to $\mathrm{M}$. Caloin for numerous discussions and critical reading of the manuscript ; to G. Pouyet of the Centre de Recherches sur les Macromolécules (C.R.M.) of Strasbourg, and to F. Hardouin and M. F. Hachard, of the Centre de Recherches Paul Pascal of Bordeaux, for doing the calorimetry measurements; to $D$. Guillon of the C.R.M. for letting me use his calorimetry results ; to P. Seuron of the Ecole de Chimie of Strasbourg for the recrystallizations of the TBBA samples; to J. Klein for his technical assistance. Part of this work was done at the Laboratoire de Physique des Solides of the Université Louis Pasteur. I thank everyone there for their kind hospitality.

\section{References}

[1] See for instance Garland, C. W., in Physical Acoustics 7 (1970) 51.

[2] Thiriet, Y., Martinoty, P., to be published in J. Physique.

[3] Bartolino, R., Scudieri, F., Sette, D. and Slivinski, A., J. Physique Colloq. 36 (1975) C1-121.

[4] BACRI, J. C., J. Physique Colloq. 36 (1975) C1-123.

[5] Miyano, K., Ketterson, J. B., Phys. Rev. A 12 (1975) 615.

[6] Kiry, F., Martinoty, P., J. Physique Colloq. 37 (1976) C3-113.

[7] Kiry, F., Martinoty, P., Ann. Phys. 3 (1978) 369.

[8] Kiry, F., Martinoty, P., J. Physique 39 (1978) 1019.

[9] Bhattacharya, S., Sarma, B., Ketterson, J. B., Phys. Rev. Lett. 40 (1978) 1582.

[10] Jähnig, F., Brochard, F., J. Physique 35 (1974) 301.

[11] Forster, D., Lubensky, T. C., Martin, P. C., Swift, J., Pershan, P. S., Phys. Rev. Lett. 26 (1971) 1016.

[12] Garland, C. W., Eden, D., Mistura, L., Phys. Rev. Lett. 25 (1970) 1161.

[13] Mistura, L., in Critical Phenomena, ed. M. S. Green (Academic Press, New York) 1971, p. 563.

[14] Nishigaki, K., J. Phys. Soc. Japan 45 (1978) 182.

[15] Kawasaki, K., Phys. Lett. 31A (1970) 165 ; see also Hohenberg, P. C., in Critical Phenomena, ed. M. S. Green (Academic Press, New York) 1971, p. 285.

[16] Imura, H., Okano, K., Chem. Phys. Lett. 19 (1973) 387.

[17] Albertini, G., Corinaldesi, M., Mazkedian, S., Melone, S., Ponzi-Bossi, M. G., Rustichelli, F., Solid State Commun. 24 (1977) 433.
[18] Als-Nielsen, J., Birgeneau, R. J., Kaplan, M. C., Litster, J. D., Safinya, C., Phys. Rev. Lett. 39 (1977) 352; see also LitsTeR et al., J. Physique Colloq. 40 (1979) C3-339.

[19] Imura, H., Okano, K., Chem. Phys. Lett. 17 (1972) 111.

[20] Stinson, T. W., Litster, J. D., Phys. Rev. Lett. 30 (1973) 688.

[21] Schnur, J. M., Sheridan, J. P., Fontana, M., in the Proceedings of the International Conference on Liquid Crystals, Bangalore (1973). Pramaha Supplement no 1 (1975).

[22] Deloche, B., Charvolin, J., Liebert, L., Strzelecki, L., J. Physique Colloq. 36 (1975) C1-21.

[23] Torza, S., Cladis, P., Phys. Rev. Lett. 32 (1974) 1406.

[24] Cabanne, B., Clark, W. G., Solid State Commun. 13 (1973) 129.

[25] Armitage, D., Price, F. P., Mol. Cryst. Liq. Cryst. 38 (1977) 229.

[26] Barral, E. M., Porter, R. S., Johnson, J. F., J. Phys. Chem. 71 (1967) 895.

[27] Bahadur, B., Mol. Cryst. Liq. Cryst. 35 (1976) 83.

[28] Eden, D., Garland, C. W., Williamson, R. C., J. Chem. Phys. 58 (1973) 1861.

[29] Candau, S., Martinoty, P., Zana, R., J. Physique Lett. 36 (1975) L-13 ;

see also NagaI, S., et al., Mol. Cryst. Liq. Cryst. 31 (1975) 243.

[30] Castro, C. A., Hikata, A., Elbaum, C., Phys. Rev. A 17 (1978) 353.

[31] Charvolin, J., Deloche, B., J. Physique Colloq. 37 (1976) C3-69;

see also DeLoche, B., Thesis Orsay (1978). 
[32] Taylor, T. R., Arora, S. L., Fergason, J. L., Phys. Rev. Lett. 25 (1970) 722.

[33] Rao, K. V. S., Hwang, J. S., Freed, J. H., Phys. Rev. Lett. 37 (1976) 515.

[34] Gulari, E., Chu,'B., J. Chem. Phys. 62 (1975) 798.
[35] Koren, G., Phys. Rev. A 13 (1976) 1177.

[36] Jähnig, F., J. Physique 36 (1975) 315.

[37] Nagai, S., Martinoty, P., Candau, S., J. Physique 37 (1976) 769.

[38] Matsushita, M., Phys. Lett. 66A (1978) 507. 